\title{
Validated tool predicts survival of men taking abiraterone after docetaxel
}

Enormous progress has been made in the treatment of castration-resistant prostate cancer (CRPC) over the past decade and, as a result, updated prognostic models are needed that reflect the extended survival associated with new therapies. Investigators at the Institute of Cancer Research in the UK, led by Johann de Bono, have externally validated a prognostic model for men treated with second-line abiraterone.

The model was created using data from the COU-AA-301 phase III trial, for which men with metastatic CRPC who had failed docetaxel-based chemotherapy were randomized to receive either abiraterone or placebo. Now, de Bono and colleagues have validated the prognostic tool in an independent cohort of 94 men who received abiraterone after docetaxel at their institution between 2006 and 2013, outside of a clinical trial.
Patient stratification-based on six baseline clinical parameters (ECOG performance status of 2; presence of liver metastases; time from start of hormone therapy to abiraterone treatment of $\leq 36$ months; low albumin; high alkaline phosphatase; high lactate dehydrogenase)-was predictive of overall survival in this cohort, with an AUC of 0.71 . Researchers also tested the tool in men who received abiraterone before chemotherapy $(n=65)$, but found it was less accurate in this population (AUC 0.61). Prospective evaluation and clinical implementation are hoped to follow.

Sarah Payton

Original article Ravi, P. et al. External validation of a prognostic model predicting overall survival in metastatic castrate-resistant prostate cancer patients treated with abiraterone. Eur. Urol. doi:10.1016/j.eururo.2014.03.020 